\title{
Comparison of Emergency Medical Services among European Union member states: A Healthcare Professionals Associations Survey
}

\author{
S. Stojanović Stipić ${ }^{1}$, M. Peric ${ }^{2}$, L. Rosini Gajsak ${ }^{3}$, T. Pavic ${ }^{4}$ A. Baric ${ }^{5}$, I. Raguz ${ }^{6}$
}

${ }^{1}$ Department of Anaesthesiology and Intensive Care Medicine, University Hospital Center - Split (Croatia), ${ }^{2}$ Department of Clinical Cytology, University Hospital Center - Osijek (Croatia), ${ }^{3}$ Department of Biological Psychiatry, Neuropsychiatric Hospital "Dr. Ivan Barbot" - Popovaca (Croatia), "Department of Internal Medicine, Clinical Hospital Center "Sestre milosrdnice"- Zagreb (Croatia), 5Department of Anaesthesiology and Intensive Care Medicine, University Hospital Center - Zagreb (Croatia), ${ }^{6}$ Department of ENT-Head and Neck Surgery, University Hospital "Sveti Duh" - Zagreb (Croatia)

Background and Goal of Study: Emergency Medical Units within the hospitals provide treatment and care of urgent patients regardless of the referral diagnosis, emerge with the integration of emergency hospital services in one place. Due to the particular diversities of organizations and functioning of emergency services within the Croatian healthcare system, our aim was to research and systematize information on the functioning of the emergency medical services in the European Union.

Materials and methods: Committee for International Cooperation of the Croatian Medical Chamber conducted a comprehensive medical emergency survey including 15 European Union (EU) member states. The questions were answered by representatives of the Czech Republic, Denmark, Germany, Austria, Hungary, Italy, Lithuania, Romania, Slovakia, Slovenia, Sweden, Estonia, Greece , Bulgaria and Malta. Questions in the survey were related to the composition of emergency care teams, the way of organizing emergency medical service in local communities, the implementation models of emergency aid at primary and secondary levels, triage scales as well as patient involvement in emergency medical assistance.

Which health care professionals provide emergency medical care by arriving in ambulance car on the intervention site?
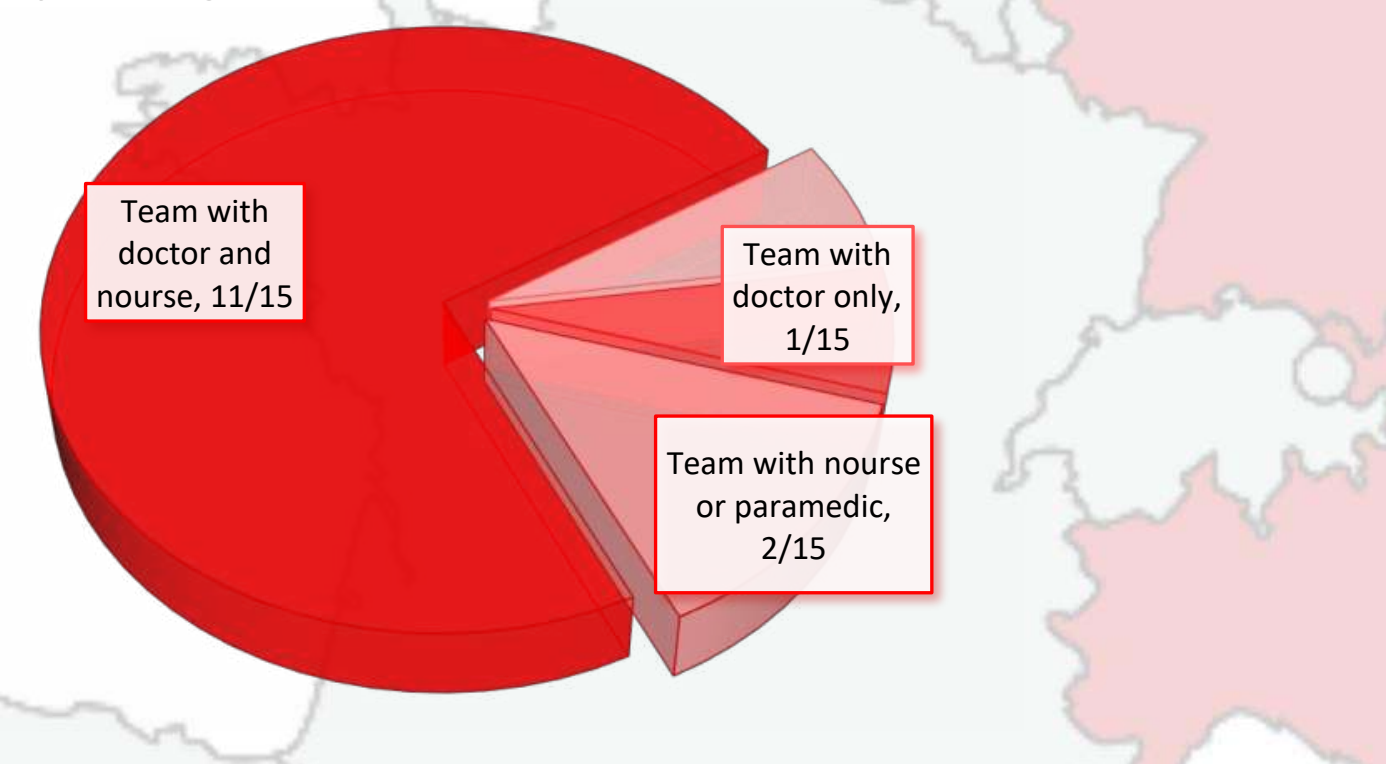

Is Emergency health care service „24 hours/7 days” in your country organized by the local government at the level of primary care?

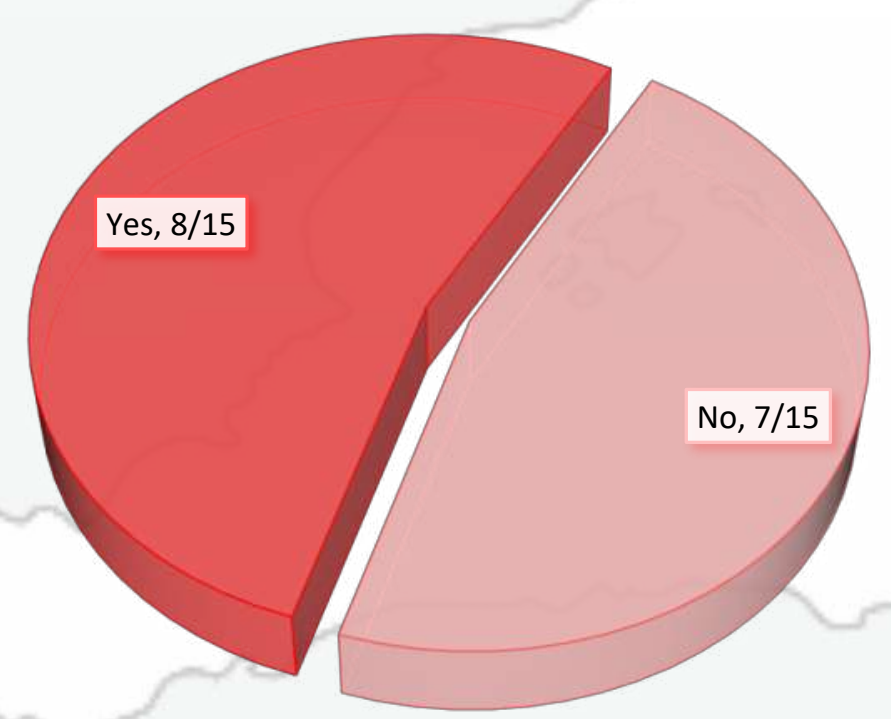

Results and discussion: Organization of emergency medical services between different EU member states mainly include a medical doctor and a registered nurse in an emergency ambulance car; in the emergency medical units patients are triaged by registered nurses; and afterwards exemined and treated by residents and specialists of internal medicine, anesthesiology and surgery. The need of specific triage scales is not uniform between different countries. General practitioners are not equally involved in the treatment of emergency patients, in $26 \%$ of countries they are not obliged to participate in the management of emergency patients, in the rest the mechanisms vary considerably. Nearly $80 \%$ of countries reported misuse of IEHM for the purpose of avoiding general practitioners, but controling this trend.

Conclusion: Most of the surveyed countries have organized Integrated Emergency Hospital Units with a few exceptions. Implementation models of emergency medical services are very divergent between surveyed countries and mostly not synchronized at national levels. Moreover, within the each country we also detected individual approaches on a regional basis or between different healthcare institutions.

Measures to discourage patients to speed up their medical treatment and/or possibly pass by their doctor

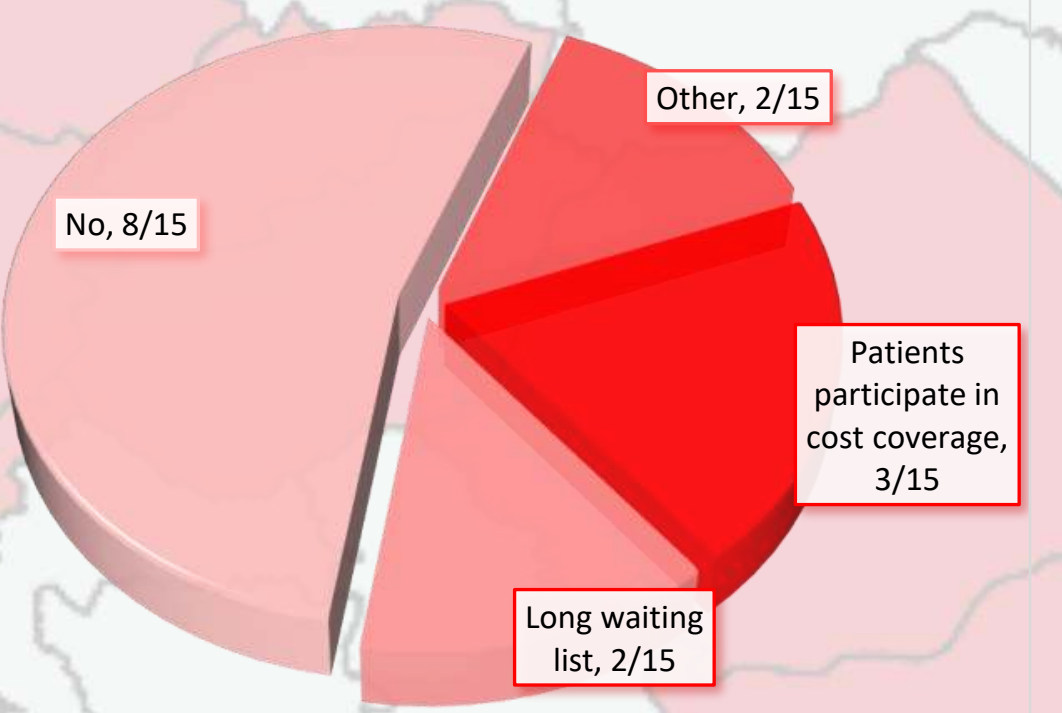

Is it possible for the patient seeking help at the ER to be sent home without being examined by MD because of the assumed banality of her/his status? 